\title{
Magnetic Seed Localization
}

National Cancer Institute

\section{Source}

National Cancer Institute. Magnetic Seed Localization. NCI Thesaurus. Code C159610.

A localization technique in which a magnetic seed marker implanted at the site of a lesion or disease is detected using a magnetic probe. 